\title{
Increasing the safety of minimally invasive hallux surgery-An anatomical study introducing the clock method
}

\author{
3 Q1 Francesc Malagelada, MD ${ }^{\mathrm{a}, \mathrm{b}, *}$, Miquel Dalmau-Pastor, Associate professor $^{\mathrm{b}, \mathrm{c}}$, \\ Betlem Fargues, Associate professor ${ }^{\mathrm{b}}$, Maria Cristina Manzanares-Céspedes, $\mathrm{PhD}^{\mathrm{b}}$, \\ Fernando Peña, $\mathrm{MD}^{\mathrm{d}}$, Jordi Vega, $\mathrm{MD}^{\mathrm{b}, \mathrm{e}}$

\footnotetext{
a Foot and Ankle Unit, Orthopaedic and Trauma Surgery, Heatherwood and Wexham Park Hospitals, Frimley Health NHS Trust, Ascot, Berkshire, UK

${ }^{\mathrm{b}}$ Laboratory of Arthroscopic and Surgical Anatomy, Human Anatomy and Embryology Unit, Department of Pathology and Experimental Therapeutics, Faculty

of Medicine and Health Sciences, University of Barcelona, Barcelona, Spain

${ }^{\mathrm{c}}$ Faculty of Health Sciences at Manresa, University of Vic-Central University of Catalonia, Manresa, Barcelona, Spain

${ }^{\mathrm{d}}$ Department of Orthopaedic Surgery, Foot and Ankle Unit, University of Minnesota, Minneapolis, MN, USA
} \\ e Foot and Ankle Unit, Hospital Quiron Barcelona, Barcelona, Spain
}

\section{A R T I C L E I N F O}

\section{Article history:}

Received 16 June 2016

Received in revised form 24 October 2016

Accepted 6 November 2016

Available online $\mathrm{xxx}$

\section{Keywords:}

Hallux valgus

Hallux rigidus

Anatomy

Minimally invasive surgery

Arthroscopy

latrogenic nerve injury

Cadaveric study

\section{A B S T R A C T}

Background: The purpose of this study is to describe a simple and reproducible method to localize the neurological structures at risk and to describe a safe zone for hallux minimally invasive surgery (MIS) procedures.

Methods: Ten fresh-frozen cadaveric feet were dissected to identify the dorsomedial digital nerve (DMDN) and the dorsolateral digital nerve (DLDN) of the first toe. Axial sections were performed at the sites of metatarsal osteotomies. We documented the position of the nerves with respect to the extensor hallucis longus (EHL) tendon using a clock method superimposed on the axial section

Results: The DMDN was found at an average of $26.2^{\circ}$ medial to the medial border of the EHL tendon. (SD 11.26 , range $14.5-45.5$ ), whereas the average distance of the DLDN was $32.3^{\circ}$ lateral to the medial border of the EHL tendon. (SD 6.29, range 13.5-40).

Conclusions: Using the clock method the DMDN and DLDN were found consistently between 10 o'clock and 2 o'clock in either right and left feet. The clock method may facilitate avoiding the area where these nerves are located serving as a valuable tool in minimally invasive foot surgery.

๑ 2016 European Foot and Ankle Society. Published by Elsevier Ltd. All rights reserved.

\section{Introduction}

The increasing popularity of percutaneous or minimally invasive surgery (MIS) of the foot has led to a number of studies reporting its benefits but also its inherent risks when compared to open surgery [1-4]. One of the feared risks of MIS is iatrogenic damage to neurovascular and tendinous structures. One cadaveric study has deemed MIS forefoot surgery safe and has reported

\footnotetext{
* Corresponding author at: Foot and Ankle Unit, Orthopaedic and Trauma Surgery, Heatherwood and Wexham Park Hospitals, Frimley Health NHS Trust, Ascot, Berkshire, UK.

E-mail addresses: fmalagelada@gmail.com (F. Malagelada), mikeldalmau@gmail.com (M. Dalmau-Pastor), betlemfarguespolo@gmail.com (B. Fargues), mcmanzanares@ub.edu (M.C. Manzanares-Céspedes), pena0013@umn.edu (F. Peña), jordivega@hotmail.com (J. Vega).
}

minimal risk, which is consistent with clinical studies in the literature, although no anatomical references were provided [5].

MIS for hallux valgus was described amongst the first techniques in the development of this discipline. It is performed with variable incidence depending on the geographic location across the globe. To be applied successfully, operative techniques must be described in detail while using reproducible reference landmarks. When performing these procedures the surgeon is usually positioned at the distal end of the table, thus the patient's feet are not in a plantigrade position i.e. that used in anatomic textbooks. The classically described reference landmarks are difficult to apply during the surgical procedure given the position of the surgeon with respect to the foot. An effort should be made to relate the results found in the anatomy lab to the operating room.

The purpose of this study is to describe a simple and reproducible method to localize the neurological structures at 
risk during percutaneous hallux surgery. Consequently a safe zone for the incision and use of percutaneous instruments is described.

\section{Methods}

We followed the surgical descriptions of De Prado et al. [6] to simulate the skin incision sites and instrument entry portals corresponding to hallux valgus (HV) and hallux rigidus (HR) pathology. The surgical procedures evaluated in this study include bunionectomy (i.e. cheilectomy), distal first metatarsal osteotomies (DFMO), and lateral release. The skin incision was considered to be at a distance of $1.5 \mathrm{~cm}$ proximal to the metatarsophalangeal joint (MTPJ), which is the entry point for the bunionectomy and DFMO procedures.

Ten unpaired feet (five left and five right), from Caucasian fresh frozen voluntary donor specimens were carefully dissected in the anatomy laboratory. The specimens included 5 men and 5 women with a median age of 80 (range, 53-95) years. All feet were amputated at the level of the distal tibia. Specimens were excluded if they presented any degree of deformities, ulcers or surgical incisions from a history of foot or ankle surgery.

The course of the dorsolateral digital nerve (DLDN) of the first toe, the dorsomedial digital nerve (DMDN), and its branches were identified after creating a window of approximately $10 \times 5 \mathrm{~cm}$ that only affected the skin, without manipulation of the nerves. The location of the window was at the level of the first metatarsal (M1) and extended from the first tarsometatarsal joint to the distal phalanx of the hallux and from the medial aspect of the sole to the second ray. Following skin dissection the nerves and terminal branches could be visualized directly within this window (Fig. 1). The plantar medial branch (PMB) of the dorsomedial digital nerve described by Solan et al. was recorded when present [7]. The origin of this branch in relation to the MTPJ was measured. The distance from the extensor hallucis longus (EHL) tendon to the DLDN was also recorded (Fig. 2).

The foot was subsequently frozen in order to fix the soft tissues and neural structures in their anatomic position. Once frozen an axial section of the foot was performed with a diamond band saw (Exakt Advanced Technologies GmbH, Norderstedt, Germany) at the point corresponding to the aforementioned site for osteotomy/ bunionectomy ( $1.5 \mathrm{~cm}$ proximal to the MTPJ) (Fig. 3 ). To localize the joint correctly from the surface, green 21 gauge $40 \mathrm{~mm}$ (1.5 in.)

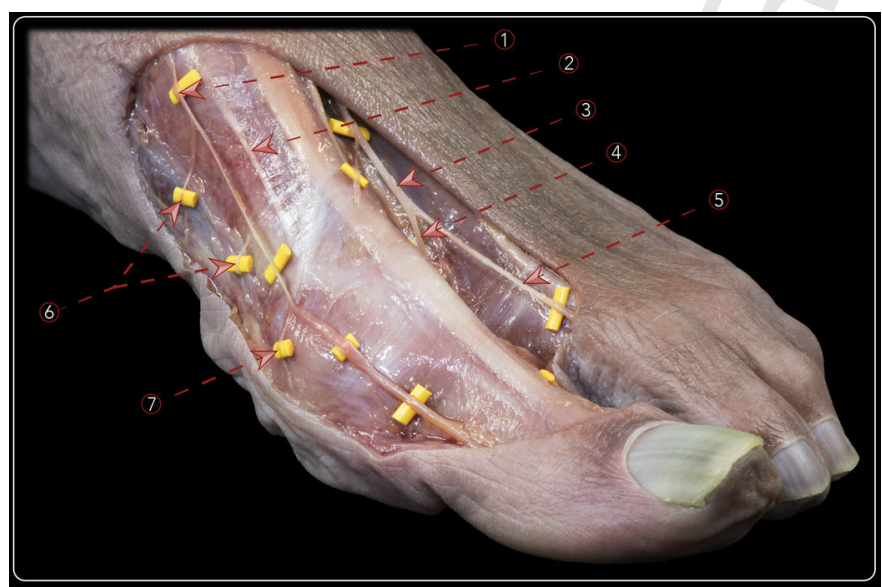

Fig. 1. Dissection of a left foot after performing a skin window to show the course of the dorsomedial digital nerve (DMDN) of the first toe and its plantar medial branch (PMB). (1) DMDN of the first toe (branch of the superficial peroneal nerve). (2) Extensor hallucis capsularis (tendinous slip of the extensor hallucis longus). (3) Deep peroneal nerve. (4) Dorsolateral digital nerve (DLDN) for the first toe. (5) DMDN for the second toe. (6) Medial cutaneous branches of the DMDN. (7) PMB of the DMDN at the level of the first metatarsophalangeal joint.

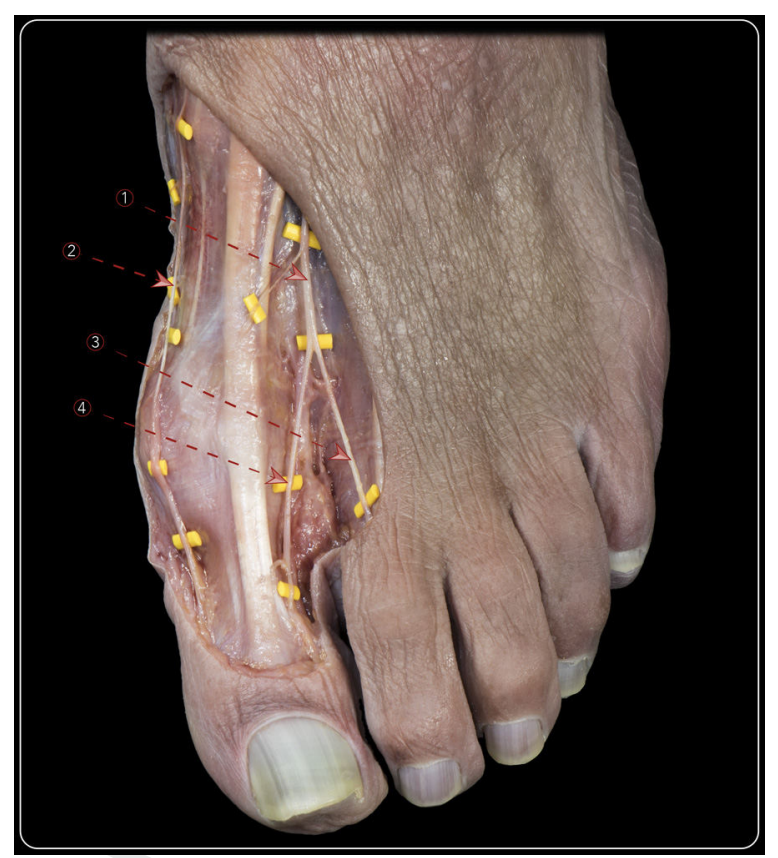

Fig. 2. Dorsal view of a dissection of the first intermetatarsal space showing the course of the dorsolateral digital nerve (DLDN) of the first toe. (1) Deep peroneal nerve. (2) Dorsomedial digital nerve (DMDN) of the first toe (branch of the superficial peroneal nerve). (3) DMDN for the second toe. (4) DLDN for the first toe.

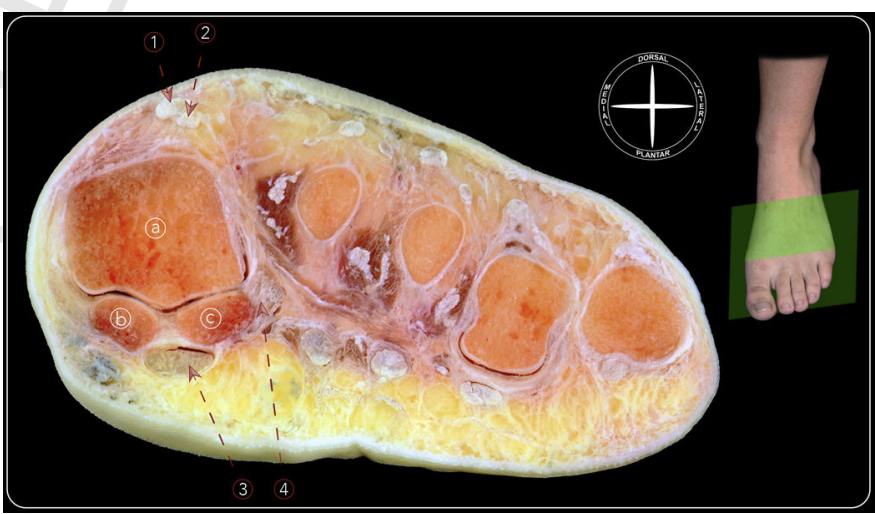

Fig. 3. Axial cross section at the level of the first metatarsal head, $1.5 \mathrm{~cm}$ from the first metatarso-phalangeal joint. (a) Head of the first metatarsal. (b) Medial sesamoid. (c) Lateral sesamoid. (1) Extensor hallucis longus tendon. (2) Extensor hallucis brevis tendon. (3) Flexor hallucis longus tendon. (4) Adductor hallucis tendon.

needles were inserted intraarticularly in the MTPJ. Likewise, needles were used to fix the nerves down to the soft tissues to avoid displacement during the sawing process.

Once the section was made the foot was held in a plantigrade position on a flat surface. Using the medial border of the EHL as a reference point corresponding to $0^{\circ}$, measurements of the location of the DMDN and the DLDN were obtained using a calibrated goniometer with $1^{\circ}$-increment scale. A line perpendicular to the floor was used as a reference to align the goniometer. The axis of the goniometer was positioned at the mid point of the vertical diameter of the metatarsal head. All the measurements were obtained by two independent, experienced anatomists.

A clock model was placed on the surface of the cross section performed at the first ray. Following the line perpendicular to the floor that connected with the medial border of the EHL the clock was positioned so that the dorsal end of the line would correspond 
to 12 o'clock, and the plantar one to 6 o'clock. The 3 o'clock position and 9 o'clock positions were subsequently aligned to medial and lateral respectively in a right foot. In a left foot 3 o'clock was lateral and 9 o'clock medial.

\subsection{Statistical analysis}

Each of the measurements performed by two observers were assessed for inter-observer reliability through intraclass correlation coefficient (ICC) and Cronbach's alpha coefficient. A value above 0.8 was considered to have reliable consistency and above 0.9 excellent consistency, and a $95 \%$ confidence interval (CI) for ICC was given. Results were obtained using the statistical program SPSS version 19 (IBM, US).

\section{Results}

\subsection{Anatomy of the dorsomedial digital nerve of the hallux}

The DMDN courses along the length of the M1 and the proximal phalanx of the hallux over their dorsomedial aspect. It is in contact with the M1 and proximal phalanx throughout its entire length and it is at risk when performing osteotomies of the M1 or proximal phalanx of the hallux. The PMB is a constant structure. On average, it branches off at a point $2.18 \mathrm{~cm}$ proximal to the $1 \mathrm{st}$ MTP joint (SD 0.52, range 1-3) (Fig. 1).

\subsection{Location of the DMDN and DLDN at the sites of skin incisions}

The DMDN was found at an average of $26.2^{\circ}$ medial to the medial border of the EHL. (SD 11.26, range 14.5-45.5). The DLDN was $32.3^{\circ}$ lateral to the medial border of the EHL in average (SD 6.29 , range 13.5-40) (Table 1 ).

The inter-observer reliability coefficients showed a Cronbach's alpha of 0.986 and 0.949 , as well as an ICC of 0.975 (95\% CI: $0.903-$ $0.994)$ and 0.954 (95\% CI: 0.813-0.989) respectively for the DMDN and DLDN, which demonstrate excellent inter-observer reliability.

Distance from the EHL to the DLDN was found to be an average of $0.39 \mathrm{~cm}$ (SD 0.19, range $0.1-0.6$ ).

Using the clock method the DMDN was found in all cases

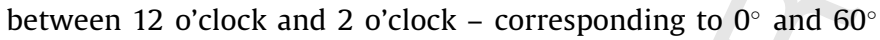
respectively - in a right foot and between 10 o'clock and 12 o'clock in a left foot - corresponding to $300^{\circ}$ and $360^{\circ}$ (dorsomedial aspect of the hallux). In contrast the DLDN was found consistently between 10 o'clock and 12 o'clock in a right foot and 12 o'clock and 2 o'clock in a left foot (dorsolateral aspect of the hallux) (Fig. 4).

\section{Discussion}

Both the dorsomedial digital nerve (DMDN) and the dorsolateral digital nerve (DLDN) are at risk of iatrogenic injury when performing hallux valgus and rigidus surgeries. In the case of MIS, the neurological structures are neither visualized nor dissected and therefore a sound knowledge of their course is paramount to avoid any damage. This study aims to serve as an intraoperative guide while describing safety zones in which to place the incisions for percutaneous surgery.

The proposed clock method for foot and ankle MIS, has been developed following a similar method popularized in knee and shoulder surgery [8-11]. During MIS the surgeon needs to recognize the location of anatomical structures without direct visualization. Hence, the clock method is ideal in these circumstances, allowing the surgeon to mentally place an imaginary clock over the first ray of the operated foot and to position anatomical structures around the clock as a reference.

Surface anatomy is helpful in some instances when anatomical landmarks are easily palpable as is the case of the MTPJ or the extensor hallucis longus tendon. However, the neurovascular structures that surround the first ray are not usually found by palpation. On the lateral side the DLDN and the first dorsal metatarsal artery are at risk when performing a lateral release of the hallux. An incision is made lateral to the extensor tendon where the nerve runs next to it. To avoid nerve damage, some authors [6] advise to insert the blade parallel to the extensor tendon and only when it is deep into the plantar aspect of the great toe, to rotate it $90^{\circ}$ to cut laterally. We measured the distance between the DLDN and the EHL tendon to be of $0.39 \mathrm{~cm}$ on average. This reference must be kept in mind when performing MIS surgery and the incision must be placed as close as safely possible to the EHL tendon to avoid injury to the nerve. Blunt dissection down to bone is also recommended as in some cases the nerve can be as close as $1 \mathrm{~mm}$ to the EHL tendon.

The medial aspect of the toe is the territory for several surgical procedures of the hallux. At $1.5 \mathrm{~cm}$ proximal to the MTPJ - where most incisions are placed - the course of the DMDN was found to be between 12 and 2 o'clock in all right-sided specimens (10 and

Table 1

Specimen characteristics with first toe dorsomedial digital nerve (DMDN) and dorsolateral digital nerve (DLDN) positions according to the clock method described.

\begin{tabular}{|c|c|c|c|c|c|c|c|c|c|}
\hline \multirow[t]{2}{*}{ Specimen } & \multirow[t]{2}{*}{ Side } & \multirow[t]{2}{*}{ Gender } & \multirow[t]{2}{*}{ Age } & \multicolumn{3}{|c|}{ DMDN position around the clock ${ }^{\mathrm{a}}$} & \multicolumn{3}{|c|}{ DLDN position around the clock $\mathrm{k}^{\mathrm{a}}$} \\
\hline & & & & Observer1 & Observer2 & Average & Observer1 & Observer2 & Average \\
\hline 1 & Left & 우 & 53 & 17 & 17 & 17 & 29 & 28 & 28.5 \\
\hline 2 & Left & q & 95 & 32 & 34 & 33 & 35 & 35 & 35 \\
\hline 3 & Left & 우 & 87 & 28 & 32 & 30 & 42 & 38 & 40 \\
\hline 4 & Right & $\hat{o}$ & 88 & 42 & 44 & 43 & 28 & 32 & 30 \\
\hline 5 & Right & q & 78 & 22 & 22 & 22 & 13 & 14 & 13.5 \\
\hline 6 & Right & ô & 73 & 45 & 46 & 45.5 & 22 & 18 & 20 \\
\hline 7 & Right & $\hat{o}$ & 83 & 25 & 22 & 23.5 & 33 & 28 & 30.5 \\
\hline 8 & Left & $\hat{\sigma}$ & 70 & 15 & 14 & 14.5 & 34 & 38 & 36 \\
\hline 9 & Right & $\hat{0}$ & 81 & 19 & 14 & 16.5 & 32 & 34 & 33 \\
\hline 10 & Left & 우 & 79 & 18 & 16 & 17 & 30 & 34 & 32 \\
\hline \multirow[t]{4}{*}{ Median } & & & 80 & Average & & 26.2 & Average & & 32.3 \\
\hline & & & & SD & & 11.26 & SD & & 6.29 \\
\hline & & & & ICC & & 0.975 & ICC & & 0.954 \\
\hline & & & & Cronbach's & & 0.986 & Cronbach's & & 0.949 \\
\hline
\end{tabular}

SD: standard deviation. ICC: intraclass correlation coefficient.

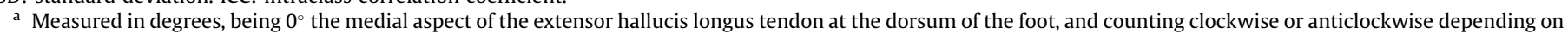
the laterality. 


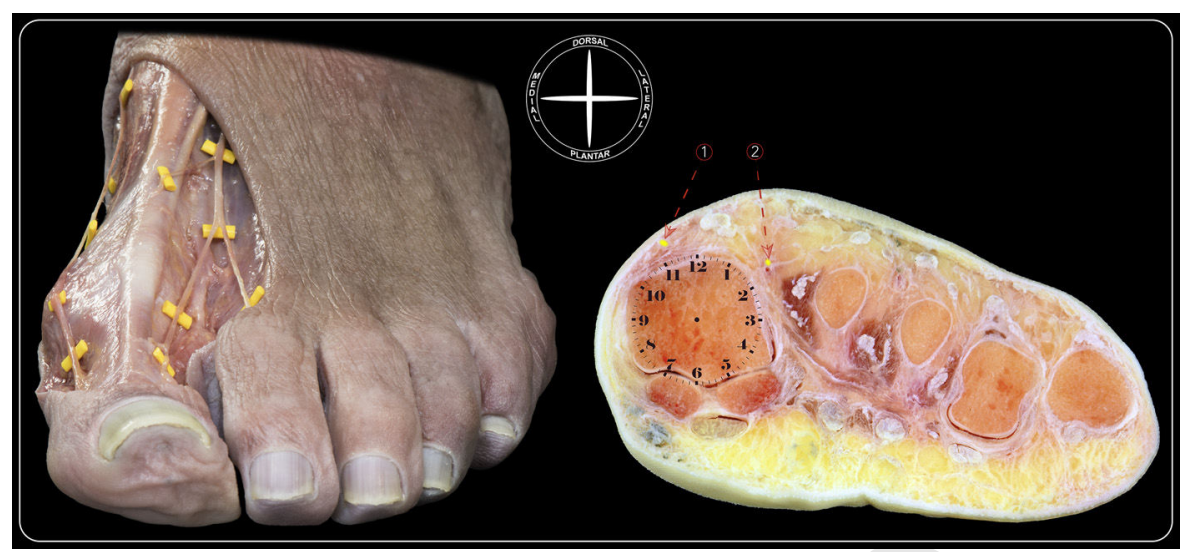

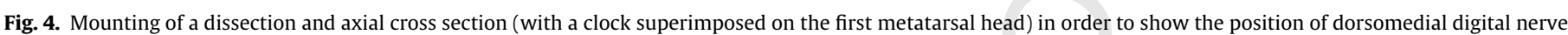

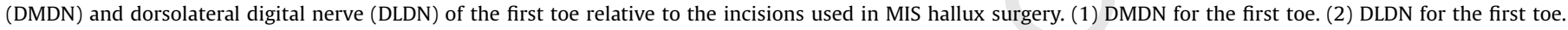

12 o'clock for left-sided ones). At this same point, the DLDN was found between 10 and 12 o'clock in right-sided feet (12 and 2 o'clock for the left-sided feet). A previous study found the PMB to arise at $20.9 \mathrm{~mm}$ proximal to the MTP joint which highly correlates with our results $(21.8 \mathrm{~mm})$ [7]. Both the DMDN and the PMB are at risk during medial skin incisions and therefore we recommend those to avoid the interval between 10 o'clock and 2 o'clock in both right or left feet. In order to avoid injury to the PMB, recommendations were made to place the incision for open hallux surgery mid-medial instead of dorsomedial [7]. One of the advantages of MIS surgery is that this branch is at lower risk due to the use of a smaller incision.

Other anatomical structures at risk of injury during hallux MIS surgery include vascular and tendinous components. We have not included them in our study as they have been described in detail in previous studies and they are more predictably localized [5,12]. The major blood supply to the metatarsal head enters through a plexus located at the plantar-lateral aspect of the metatarsal neck just proximal to the capsular attachment. This supply could be damaged while performing a Chevron osteotomy and could result in osteonecrosis of the first metatarsal head. The general advice predicated by anatomical and clinical studies is to perform the osteotomy with a long plantar arm exiting well proximal to the capsular attachment [12]. A cadaveric study in which a MIS Chevron osteotomy was performed found no injury to the capsule and soft tissue sleeve around the first metatarsal head, and therefore concluded that no damage was caused to the arterial plexus [5]. In the same study no tendon injuries were demonstrated. A precise technique is important to avoid tendon damage with the burr by keeping the tip close to the far cortex instead of proud and by holding the joint in the position that takes the tension off the tendon at risk while performing the osteotomy.

The anatomic references provided are useful for a number of osteotomies and other surgical procedures performed for hallux valgus or rigidus. Those include bunionectomy, distal first metatarsal osteotomies (Reverdin-Isham or Chevron types), cheilectomy of the first MTPJ, and wedge osteotomy of the first metatarsal. The site for the axial section and the subsequent nerve measurements was chosen according to the previously published descriptions of the relevant surgical techniques [6]. For bunionectomy, Reverdin-Isham osteotomy, cheilectomy and wedge osteotomy the incision is described at the plantar border of the medial aspect of the first metatarsal, just proximal to the medial sesamoid bone. For the Chevron osteotomy the incision is made a few millimetres dorsal to the previously described one. The medial cortical perforation is carried out at a distance of $1-1.5 \mathrm{~cm}$ proximal from the distal metatarsal articular surface. Since the surgical techniques mentioned are consistently performed at a distance of $1.5 \mathrm{~cm}$ proximal to the MTPJ, this was considered the more clinically relevant level to obtain the nerve measurements.

The results of this study can also be useful during placement of the portals in arthroscopy of the first MTPJ. The two main arthroscopic portals (dorsomedial and dorsolateral) are located at the level of the joint and therefore $1.5 \mathrm{~cm}$ distal from our point of measurement [13]. That being said, this minor difference along the length of the first ray does not translate in changes of nerve position using the clock method in our specimens. The anteromedial portal is generally located close to the EHL tendon and therefore it lies in the zone at risk of damaging the DMDN. According to our results the DMDN is found at an average of $26.2^{\circ}$ from the medial border of the EHL - corresponding to the 1 o'clock position - and consequently the closer to the EHL the least risk of nerve injury at the time of portal placement.

Limitations of this study include the fact that the freezing process may produce changes in the volume of the specimen's tissues. Nevertheless, these changes are minimal in frozen specimens and the process was deemed necessary to preserve the original course of the nerve during axial sectioning. A second limitation is that none of the selected specimens for the study presented pathologic hallux valgus deformity, which is one of the primary indications for the above-described surgical techniques. It still remains uncertain whether the nerve course would be affected by the underlying deformity, but this is a possibility.

To the best of our knowledge no previous anatomical study has been published that investigates the relationship of the neurological structures of the hallux for its application in MIS by using the clock method. Placing the skin incisions in the safe zone between 2 and 10 o'clock is a reproducible and reliable method for the surgeon to minimize the potential risk for nerve injury. Extra caution is recommended when performing a lateral release, as the incision lies in close proximity to the DLDN. Further studies that investigate its application in vivo in the operating room setting are warranted.

\section{Conflict of interest}

All authors declare that they have no relationships/conditions/ circumstances that present a potential conflict of interest for the preparation of this manuscript.

\section{Acknowledgements}

The authors wish to express their gratitude to the donors and to the Dissection Room and Cadaver Donation Service of the Faculty of Medicine of the University of Barcelona. 


\section{References}

[1] Bauer T, de Lavigne C, Biau D, De Prado M, Isham S, Laffenétre O. Percutaneous hallux valgus surgery: a prospective multicenter study of 189 cases. Orthop Clin North Am 2009;40:505-14, doi:http://dx.doi.org/10.1016/j. ocl.2009.05.002.

[2] Lucas Y Hernandez J, Golanó P, Roshan-Zamir S, Darcel V, Chauveaux D, Laffenêtre $\mathrm{O}$. Treatment of moderate hallux valgus by percutaneous, extraarticular reverse-L Chevron (PERC) osteotomy. Bone Joint J 2016;98-B(3):36573, doi:http://dx.doi.org/10.1302/0301-620x.98b3.35666.

[3] Oliva F, Longo UG, Maffulli N. Minimally invasive hallux valgus correction Orthop Clin North Am 2009;40:525-30, doi:http://dx.doi.org/10.1016/j. ocl.2009.06.005.

[4] Redfern D, Vernois J, Legré BP. Percutaneous surgery of the forefoot. Clin Podiatr Med Surg 2015;32:291-332, doi:http://dx.doi.org/10.1016/j. cpm.2015.03.007.

[5] Dhukaram V, Chapman AP, Upadhyay PK. Minimally invasive forefoot surgery: a cadaveric study. Foot Ankle Int 2012;33:1139-44, doi:http://dx.doi.org/ 10.3113/FAI.2012.1139.

[6] De Prado M, Ripoll P, Golanó P. Minimally invasive foot surgery surgical techniques, indications, anatomical basis. Barcelona: About Your Health Ed. 2009.
[7] Solan MC, Lemon M, Bendall SP. The surgical anatomy of the dorsomedial cutaneous nerve of the hallux. J Bone Joint Surg Br 2001;83:250-2.

[8] Aglietti P, Giron F. Anterior cruciate ligament reconstruction for chronic injuries. In: Insall JN, Scott WN, editors. Surgery of the knee. 3rd ed. Philadelphia: Churchill and Livingstone; 2001. p. 717-87.

[9] Davidson PA, Tibone JE. Anterior-inferior (5 o'clock) portal for shoulder arthroscopy. Arthroscopy 1995;11(5):519-25.

[10] Edwards A, Bull AMJ, Amis AA. The attachments of the anteromedial and posterolateral fibre bundles of the anterior cruciate ligament part 2: femoral attachment. Knee Surg Sports Traumatol Arthrosc 2008;16:29-36.

[11] Siebold R, Ellert T, Metz S, Metz J. Femoral insertions of the anteromedial and posterolateral bundles of the anterior cruciate ligament: morphometry and arthroscopic orientation models for double-bundle bone tunnel placement-a cadaver study. Arthroscopy 2008;24:585-92, doi:http://dx.doi.org/10.1016/j. arthro.2007.12.008.

[12] Malal JJG, Shaw-Dunn J, Kumar CS. Blood supply to the first metatarsal head and vessels at risk with a chevron osteotomy. J Bone Joint Surg Am 2007;89:2018-22.

[13] van Dijk CN, Veenstra KM, Nuesch BC. Arthroscopic surgery of the metatarsophalangeal first joint. Arthroscopy 1998;14(8):851-5. 